\title{
Non-Compaction Cardiomyopathy Misdiagnosed as Dilated Cardiomyopathy
}

\author{
Hao Thai Phan \\ Department of Internal Medicine. Pham Ngoc Thach University of Medicine, Ho Chi Minh City, Vietnam.
}

\section{Corresponding Author: \\ Dr. Hao Thai Phan}

Email: phanthaihao@yahoo.com

This is an Open Access article distributed under the terms of the Creative Commons Attribution License (creativecommons.org/ licenses/by/3.0).

Received : September 15, 2017

Accepted : January 5, 2018

Published : January 15, 2018

\begin{abstract}
Background: Non-compaction cardiomyopathy is a rare cardiomyopathy, until now, not fully clarified. Diagnosis remains difficult since there are similarities with other cardiac defects, clinical manifestations are non-specific and echocardiographic criteria are not universally accepted. As a consequence diagnosis may be easily missed. We present a clinical case of previously healthy and asymptomatic boy, misdiagnosed as dilated cardiomyopathy. Case Report: A 13-year-old previously healthy boy presented with a 2 day of abdominal pain after being hit by his friend. Physical examination showed signs of congestive cardiac failure. Echocardiography and cardiac MS-CT were consistent with the diagnosis of non-compaction cardiomyopathy. Conclusion: Our purpose with this case report was to point out attention to this entity as an important and often misdiagnosed cause of dilated cardiomyopathy.
\end{abstract}

Keywords: Abdominal Pain, Dilated Cardiomyopathy, Echocardiography, Endocardium, Myocardium.

\section{Introduction}

Noncompaction cardiomyopathy or "spongy myocardium", is a rare congenital cardiomyopathy that can be diagnosed at any age. It is characterized by a thin, compacted epicardial layer and an extensive non-compacted endocardial layer, with prominent trabeculation and deep recesses that communicate with the left ventricular cavity but not with the coronary circulation [1], probably due to an arrest of compaction during intrauterine life. It can be isolated or associated with other congenital diseases. Based on echocardiographic studies, reported prevalence is between 0.014 and $1.3 \%$ in the general population. Eventually, this condition can potentially lead to chronic heart failure, life threatening ventricular arrhythmias and systemic embolic events [2].

\section{Case Report}

A 13-year-old boy presented with a two day history of abdominal pain after being hit by his friend. He was previously healthy, referring no prior cardiac or pulmonary complaints. His father died of gastric cancer at the age of 40 , his elder brother died of heart failure when he was 13 years old. He was referred from a hospital with provisional diagnosis of dilated cardiomyopathy and treated for heart failure. On admission to the Emergency Ward of our hospital, his vital signs were blood pressure: 80/48 mmHg, pulse: $78 \mathrm{bpm}, \mathrm{SpO} 297 \%$ (air room), temperature $37^{\circ} \mathrm{C}$. Patient clinically stabilized within next 24 hours and was referred to the Cardiology Ward. Physical examination showed point of maximum impulse at the sixth intercostal space and anterior axillary line, S3 and a grade III/VI holosystolic murmurs in apical; liver edge below $2 \mathrm{~cm}$ the right costal margin.

Chest X-ray demonstrated increased heart-thorax index [Fig.1]. ECG show right axis deviation, enlargement of both atria, hypertropihy of both ventricles, and ventricular premature contraction [Fig.2]. Holter ECG showed sinus 


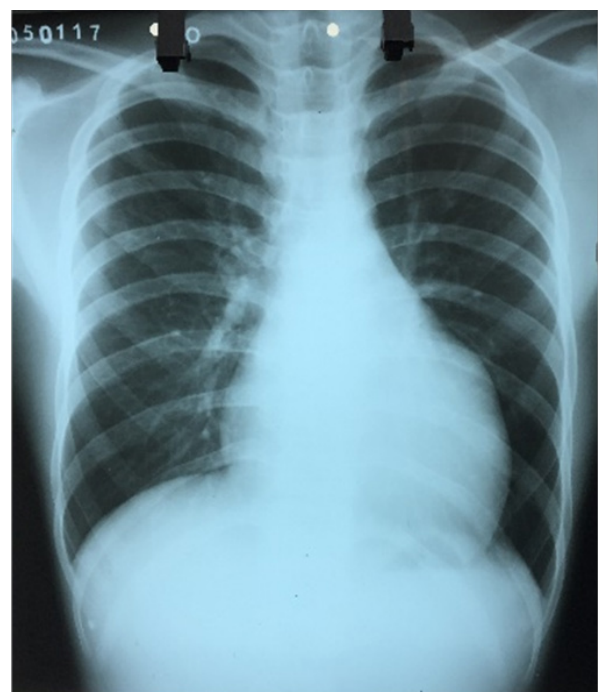

Fig.1: $P A$ Chest $X$-ray show large left ventricular trophy.

tachycardia, isolated ventricular premature contraction was $17 \%(25,219), 4$ beats fastest run $227 \mathrm{bpm}$. TSH was $1.84 \mu \mathrm{IU} / \mathrm{mL}$ (normal value 0.35-4.94 $\mu \mathrm{IU} / \mathrm{mL})$. HDL-C was low $0.75 \mathrm{mmol} / \mathrm{L}$. Liver transaminases levels and renal function tests were normal. Carotid and aortic artery Doppler ultrasound were normal. An echocardiography demonstrated exuberant thickening and trabeculation of the lateral and posterior walls of the apical half of the left ventricle with two distinct myocardial layers: a normal compact (C) epicardium and a thickened non-compact (NC) endocardium. The ratio between $\mathrm{NC}$ endocardium and $\mathrm{C}$ epicardium $>2.2$ (measured at end systole in parasternal short axis view). Left ventricle cavity was dilated and presented diffuse hypokinesis and an ejection fraction of $20 \%$. No additional abnormalities were found [Fig.3-4]. These findings were consistent with the diagnosis of noncompaction cardiomyopathy. A cardiac MS-CT 640 confirmed the diagnosis, revealing numerous proeminent trabeculae and deep intertrabecular recesses and a ratio between $\mathrm{NC}$ endocardium and C epicardium $>2,2$ [Fig 5-10].

\section{Discussion}

Non-compaction cardiomyopathy is considered a rare disease, although its exact prevalence is

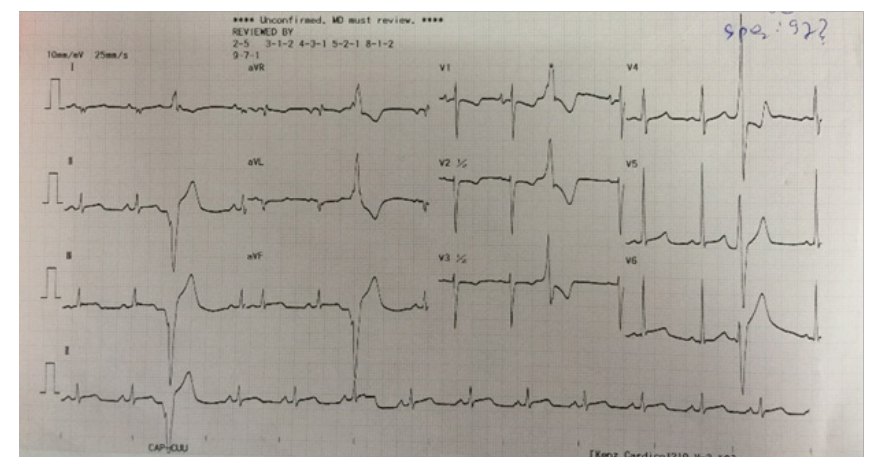

Fig.2: ECG shows sinus rhythm, premature ventricular contraction, low amplitude QRS in limb leads and left ventricular enlargement.
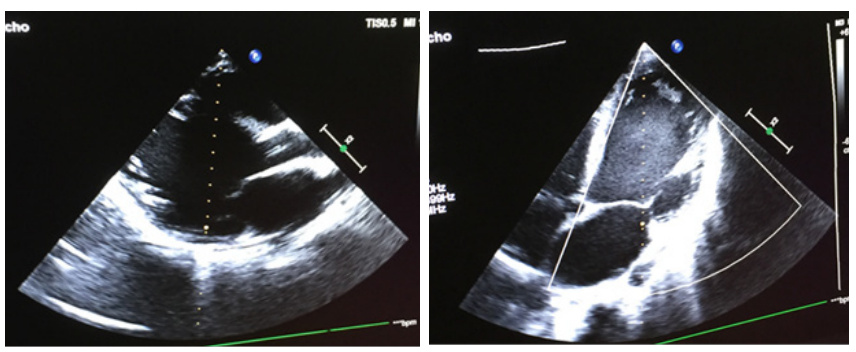

Fig.3-4: A echocardiography demonstrated exuberant thickening and trabeculation of the lateral and posterior walls of the apical half of the left ventricle with two distinct myocardial layers: a normal compact $(C)$ epicardium and a thickened non-compact (NC) endocardium. The ratio between NC endocardium and C epicardium $>2.2$ (measured at end systole in parasternal short axis view). Left ventricle cavity was dilated and presented diffuse hypokinesis and an ejection fraction of $20 \%$.

unknown. According to one of the largest series published, its prevalence was $0.014 \%$ in a group of patients referred to an echocardiography laboratory for abnormal findings or congestive heart failure [5]. Men appear to be more frequently affected, accounting for 56 to $82 \%$ of reported cases $[5,6]$. Several diagnostic criteria have been proposed in the literature [3]. In this clinical case diagnosis was primarly established by echocardiography according to the criteria defined by Jenni and colleagues. According to these authors diagnosis is based on the detection of two myocardial layers, with a normal compact (C) epicardium and a thickened non-compact (NC) endocardium. They propose a quantitative evaluation by determining 

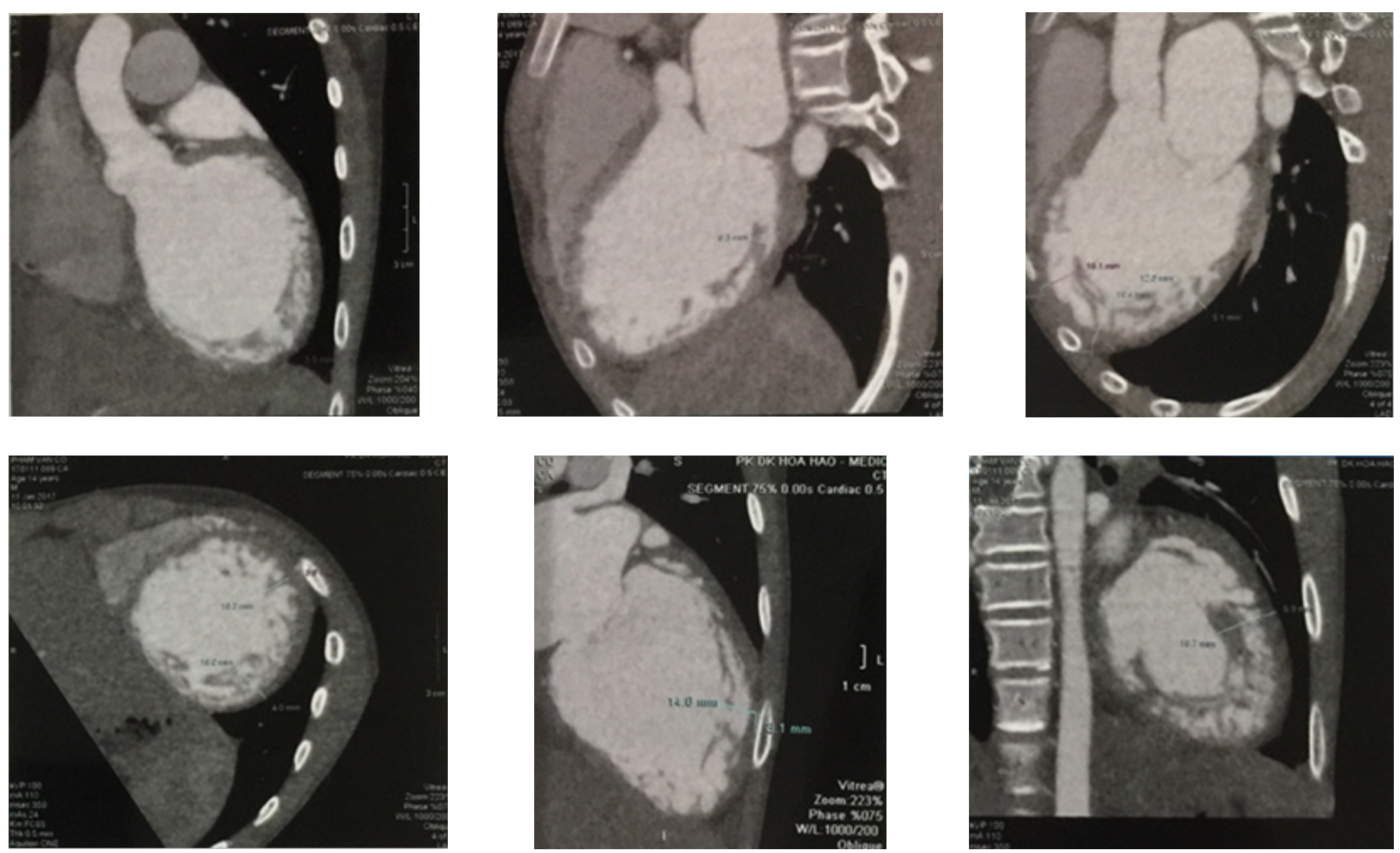

Fig.5-10: Cardiac MS-CT 640 slices confirming non-compaction cardiomyopathy. Numerous proeminent trabeculae and deep intertrabecular recesses. Two-layered myocardium, ratio non-compacted endocardium to compacted epicardium $>2.2$.

the ratio between the 2 layers $\mathrm{NC} / \mathrm{C} \geq 2$, measured at end systole in parasternal short axis view, to establishe diagnosis. This allows differentiation of the trabeculations of non-compaction cardiomyopathy from that observed with dilated cardiomyopathy and hypertropic cardiomyopathy.

\section{Conclusion}

Non-compaction cardiomyopathy is a rare and recently described congenital cardiomyopathy. Its impact on morbidity and mortality is gaining importance as it is recognized with increasing frequency. An early, reliable diagnosis is crucial and echocardiography represents a prominent role. Treatment is directed towards its most important clinical implications, which include heart failure, arrhythmias and systemic embolic events. Heart transplantation is the only definitive treatment.
Attending to the risk of cardiac sudden death, implantable cardioverter-defibrillator (ICD) may have a role in these patients.

Contributors: HTP wrote the manuscript, did literature search, involved in patient management and approved the final version of this article. He will act as guarantor of the study. Funding: None; Competing interests: None stated.

\section{References}

1. Chin TK, Perloff JK, Williams RG, Jue K, Mohrmann R. Isolated noncompaction of left ventricular myocardium. A study of eight cases. Circulation. 1990;82:507-513.

2. Engberding R, Bender F. Identification of a rare congenital anomaly of the myocardium by twodimensional echocardiography: persistence of isolated myocardial sinusoids. Am J Cardiol. 1984;53:17331734.

3. Jenni R, Oechslin EN, Loo van der B. Isolated ventricular non-compaction of the myocardium in adults. Heart. 2001;93:11-15. 
4. Bento J, Monteiro F, Sargento L, Vizcaino J, Monteiro J, PilarAzevedo P, etal. Isolated ventricular noncompaction: a case report. Cases Journal. 2009;2:9312.

5. Oechslin EN, Attenhofer Jost CH, Rojas JR, Kaufmann PA, Jenni R. Long-term follow-up of 34 adults with isolated left ventricular noncompaction: a distinct cardiomyopathy with poor prognosis. J Am Coll Cardiol. 2000;36:493-500.

6. Ritter M, Oechslin E, Sutsch G, Attenhofer C, Schneider J, Jenni R. Isolated Noncompaction of myocardium in adults. Mayo Clinic Proc. 1997;72:26-31. 